\title{
Performance of the High Momentum Particle Identification CsI-RICH for ALICE at CERN-LHC
}

\author{
Abraham Gallas \\ CERN PH-AIT, Geneva 231211 CH, Switzerland \\ on behalf of the ALICE/HMPID Group
}

\begin{abstract}
The ALICE High Momentum Particle Identification (HMPID) detector consists of an array of seven identical proximity focusing Ring Imaging Cherenkov counters. It has been designed to identify charged pions and kaons in the range $1<p_{t}<3 \mathrm{GeV} / \mathrm{c}$ and protons in the range $2<p_{t}<5 \mathrm{GeV} / \mathrm{c}$. The total active area of this detector is about $11 \mathrm{~m}^{2}$ and represents the largest scale application of CsI photocathodes to date. The detector performance, as being evaluated in test beam at CERN, will be discussed for the first five modules.
\end{abstract}

Key words: RICH, Photocathode, CsI, Cesium iodide, Particle Identification, HMPID

PACS: 25.6, 34.8a

\section{Introduction}

ALICE (A Large Ion Collider Experiment) has been specifically optimized to study, at the $\mathrm{LHC}, \mathrm{Pb}-\mathrm{Pb}$ collisions at a center-of-mass energy per nucleon pair of $\sqrt{S_{N N}}=5.5 \mathrm{TeV}$ with a luminosity of $10^{27} \mathrm{~cm}^{-2} \mathrm{~s}^{-1}$ [1]. It is foreseen that head-on collisions between ultra-relativistic heavy nuclei will attain the extreme conditions of energy densities needed to determine the transition of hadronic matter to a short-lived state, called the Quark Gluon Plasma (QGP), where quarks are no longer confined within the nucleon. ALICE will measure and identify mid-rapidity ${ }^{1}$ hadrons, leptons and photons produced in the interaction. Its layout covers a wide range of momenta (from $100 \mathrm{MeV} / \mathrm{c}$ up to $100 \mathrm{GeV} / \mathrm{c}$ ) and will cope with the highest particle density expected of 8000 per unit of rapidity in central $\mathrm{Pb}-\mathrm{Pb}$ collisions. For charged hadrons, the particle identification (PID) involves several detectors. Covering the full barrel we have the Inner Tracking

$\overline{1-0.9}<\eta<0.9$. 


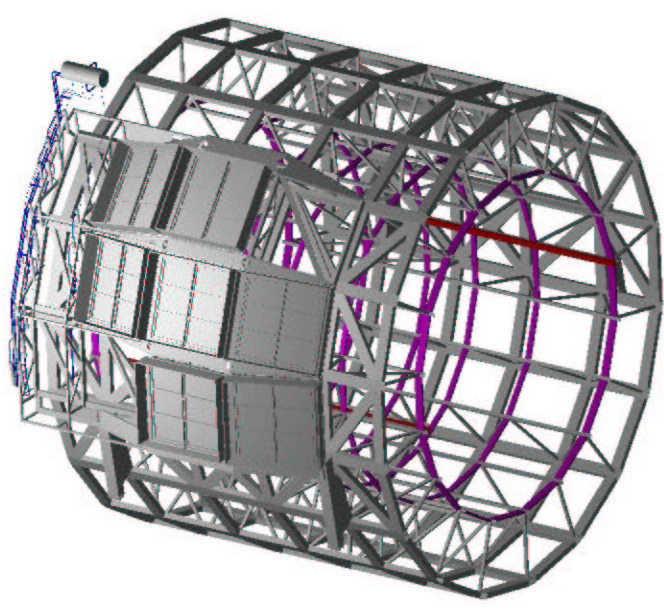

Fig. 1. View of the ALICE space frame. The HMPID modules are mounted onto a cradle located at two o'clock position.

System, the TPC (both performing $\mathrm{dE} / \mathrm{dx}$ measurements) and the Time of Flight system. Then, there is a single-arm detector, the High Momentum PID (HMPID), devoted to the identification of high transverse momentum, $p_{t}$, charged particles.

\section{The HMPID layout}

The physics motivation for the HMPID detector is the determination of inclusive particle ratios and transverse momentum spectra as well as the measurement of momentum correlations of the identified particles. Specifically, the aim is to identify with a $3 \sigma$ level separation charged pions and kaons in the range $1<p_{t}<3 \mathrm{GeV} / \mathrm{c}$ and protons in the range $2<p_{t}<5 \mathrm{GeV} / \mathrm{c}$, which implies a resolution of the Cherenkov angle $\theta_{c} \leq 3 \mathrm{mrad}$.

The HMPID will be operated in a high charged particle multiplicity environment with particle densities up to $100 \mathrm{~m}^{-2}$. The occupancy foreseen is $\sim 12 \%$ and the acceptance required to achieve the physics goals is $5 \%$ of the central barrel space, with pseudo-rapidity and azimuthal coverages of $-0.6<\eta<0.6$ and $57.6^{\circ}$ respectively. The interaction rates will go from $8 \mathrm{kHz}$ in $\mathrm{Pb}-\mathrm{Pb}$ to 300 $\mathrm{kHz}$ in $\mathrm{p}-\mathrm{p}$ collisions.

Given the above experimental conditions, the detector should have single photon sensitivity, i.e. a high quantum efficiency (QE), a good localization accuracy, and a low noise Front End Electronics (FEE) allowing a single $e^{-}$efficiency $>85 \%$. The solution has been a proximity focusing Ring Imaging Cherenkov (RICH) detector. The detector consists of an array of seven identical modules of $1.5 \mathrm{~m} \times 1.5 \mathrm{~m}$, arranged in a cupolalike structure and located at a radial distance of $4.7 \mathrm{~m}$ from the interaction point (Fig. 1) [2].

A detailed description of the ALICE HMPID RICH can be found in [2]-[4], here the most general features will be pointed out. The cross section of the detector is shown in Fig. 2. The momentum range covered by the HMPID is achieved by means of a $15 \mathrm{~mm}$ thick layer of $C_{6} F_{14}$ liquid radiator (refractive index $n=1.2989$ at $\lambda=175 \mathrm{~nm}$ ). Then the Cherenkov cone refracts out of the liquid radiator and expands in the proximity volume of pure $\mathrm{CH}_{4}$ reaching the CsI photocathode (PC) of an open MWPC that works as the photon detector. The chamber is operated at atmospheric pressure. The photocathode is segmented into pads ( 8 $\mathrm{mm} \times 8.4 \mathrm{~mm})$. It is coated with a 


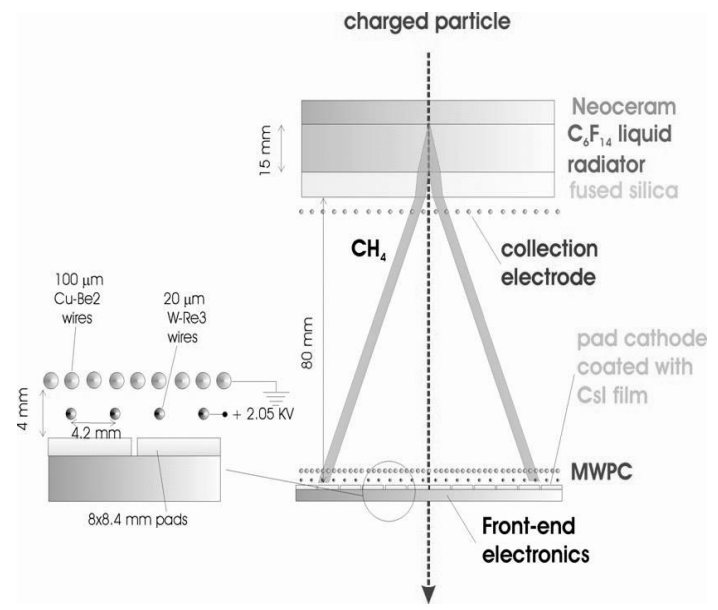

Fig. 2. Schematic cross-section of the HMPID RICH detector.

$300 \mathrm{~nm}$ photosensitive layer of CsI (in reflective mode), and is equipped with an analogue single pad readout. The sensitive gap of the MWPC is $4.45 \mathrm{~mm}$, the cathodes are grounded and a positive voltage of 2050 volts is applied to the anode wire plane providing a total gas gain of about 5 $\times 10^{4}$.

The gas and liquid systems have been designed to fulfill the requirements of high purity and safe operation. Gas purity is needed for the stability of the MWPC efficiency, specially to avoid any QE deterioration of the photocathode, due to the large hygroscopicity of the CsI. The liquid circulation system has to fill and drain the radiators vessels (8 1 each) independently, remotely and safely at a constant flow of $4 \mathrm{l} / \mathrm{h}$. A system based on gravity flow has been chosen due to its safe nature to prevent any accidental hydrostatic overpressures. Since $C_{6} F_{14}$ has a high affinity for $\mathrm{O}_{2}$ and $\mathrm{H}_{2} \mathrm{O}$, which spoils the transparency in the UV region for the Cherenkov light, a dedicated filtering station had to be built with
l3X molecular sieves [2] [5].

The front-end (FEE) and read-out $(\mathrm{R} / \mathrm{O})$ electronics are enclosed in a box on the backside of the module. The box is flushed with nitrogen and closed with a cooling panel. The total heat dissipated by one module amounts to $400 \mathrm{~W}$ and will be removed by the cooling system that delivers water at $17^{\circ} \mathrm{C}$ through the heat screen.

\section{Detector assembly and qual- ity checks}

The HMPID production can be divided in three sub-projects: the MWPC with the radiator vessels, the electronics and the CsI PCs.

\subsection{Chamber construction}

Each module contains three radiator vessels with a size of $1330 \mathrm{~mm} \times$ $413 \mathrm{~mm} \times 24 \mathrm{~mm}$. They are made of NEOCERAM ${ }^{\circledR}$, a glass-ceramic material thermally compatible with the fused silica plates used as UVtransparent windows. The MWPC is a stack of four frames made of aluminium, with a size of $1.5 \mathrm{~m} \times 1.5$ $\mathrm{m}$, holding the different wire planes. The anode-cathode (PC) gap is 2 $\mathrm{mm}$. The $20 \mu \mathrm{m}$ thick gold plated $\mathrm{W}$-Re anode wires $^{2}$, spaced $4.2 \mathrm{~mm}$ apart, are soldered on a G10 printed board with a precision better than $0.1 \mathrm{~mm}$ and a tension of $46 \pm 1 \mathrm{~g}$.

$\overline{297 \%} \mathrm{~W}$ and $3 \%$ of Re in weight. 
The second cathode plane, placed $2.45 \mathrm{~mm}$ above the anode plane, is made of $100 \mu \mathrm{m}$ thick gold plated $\mathrm{Cu}-\mathrm{Be}$ wires ${ }^{3}$, with a pitch of 2.1 $\mathrm{mm}$. It is parallel to the anode wires and stretched at a tension of $210 \pm$ $5 \mathrm{~g}$ using crimping pins. Finally the third wire plane, the collection electrode, is placed next to the radiator vessel exit window. It is made of 100 $\mu \mathrm{m}$ diameter gold plated $\mathrm{Cu}$-Be wires with a pitch of $5 \mathrm{~mm}$, at a tension of $55 \pm 1.5 \mathrm{~g}$. This wire plane, that is positive biased (400 volts), works as a collection electrode to prevent electrons released by charged particles in the proximity gap from reaching the MWPC. The proximity gap is $80 \mathrm{~mm}$. All these frames are stacked together to build a HMPID module.

The gas tightness is ensured by soft O-rings (FKM 75) placed in grooves between the chamber frames. During and after the module construction, several quality checks are performed: metrology of the different parts, wire tension measurement, leak rate measurement, HV commissioning under $\mathrm{CO}_{2}$ and $\mathrm{CH}_{4}$, and gain mapping with a ${ }^{90} \mathrm{Sr}$ source.

\subsection{Readout electronics}

The task of the HMPID front-end and readout electronics is to measure the analogue signal induced on the pads of the MWPC cathode plane. Two ASICs have been specifically developed to perform this task ${ }^{4}$ : the

$\overline{398 \%} \mathrm{Cu}$ and $2 \%$ of Be in weight.

4 Manufactured by IMEC (Leuven, Belgium) in the $0.7 \mu \mathrm{m}$ n-well CMOS
Gassiplex07-3 and the Dilogic-3 [9]. The first is a 16-channel multiplexed analogue low noise signal processor. The second is a sparse data scan digital processor that performs the zero suppression and the pedestal subtraction. All the chips needed to read out the seven modules (10080 Gassiplex and 3360 Dilogic) have been mounted on cards and tested. During the test beam validation of the HMPID modules, several noise measurements of the electronics on the detector have been made. The average on-detector noise measured for 2880 chips is less than $1000 e^{-}$ r.m.s with a dispersion in the measurement less than $50 e^{-}$.

\subsection{Photocathode fabrication}

Every module has six photocathodes with a size of $64 \mathrm{~cm} \times 40 \mathrm{~cm}(80$ $\times 48$ pads of $8 \mathrm{~mm} \times 8.4 \mathrm{~mm}$ ). The pad cathode boards are coated with CsI in a large evaporation plant at CERN, described in detail in ref [6]. It has been shown [2], [7], that the CsI QE depends on the surface quality at microscopic level of the substrate and on the handling conditions after the CsI deposition. Therefore a special procedure has been established to ensure high and reproducible QE [8], [6]. Double layer printed circuit boards (PCBs) will serve as substrate for the CsI thin film. The PCBs are specially prepared, the $\mathrm{Cu}$ pads are covered with a $7 \mu \mathrm{m}$ layer of $\mathrm{Ni}$ and then a $0.5 \mu \mathrm{m}$ of $\mathrm{Au}$. Since pollution may happen during the many

$\overline{\text { technology }}$ 
steps of the production, a special ultrasonic cleaning of the pad cathode has to be performed just before the CsI deposition. After that, the pad cathode is coated with a $300 \mathrm{~nm}$ film of CsI under a pressure of $5 \times 10^{-7}$ millibar and a temperature of $60^{\circ} \mathrm{C}$. The pad cathode is kept under vacuum at $60^{\circ} \mathrm{C}$ for $12 \mathrm{~h}$, heat treatment, to achieve the final QE.

In view of the large scale production of CsI photocathodes, the deposition plant has been equipped with a dedicated VUV scanner system to evaluate in-situ the photoemission and the response homogeneity over the full photocathode surface. The system and the measuring procedure are described in detail in [6]. The photocathode is encapsulated in a sealed protective box and kept under dry Ar flow before the extraction and during its entire lifetime to avoid moisture. Eventually it is inserted in a HMPID module using a large custom-made glove box under a controlled Ar atmosphere to avoid any exposure to ambient air.

\section{Test beam and detector per- formance}

The commissioning of the HMPID modules in the test beam is a crucial step of the validation procedure for the MWPC and for the photocathodes (PCs). The MWPC is studied in terms of operation stability (HV, rate, noise) and gain uniformity. The test beam data are also used to crosscheck and validate the results from the VUV-scanner, which is em-

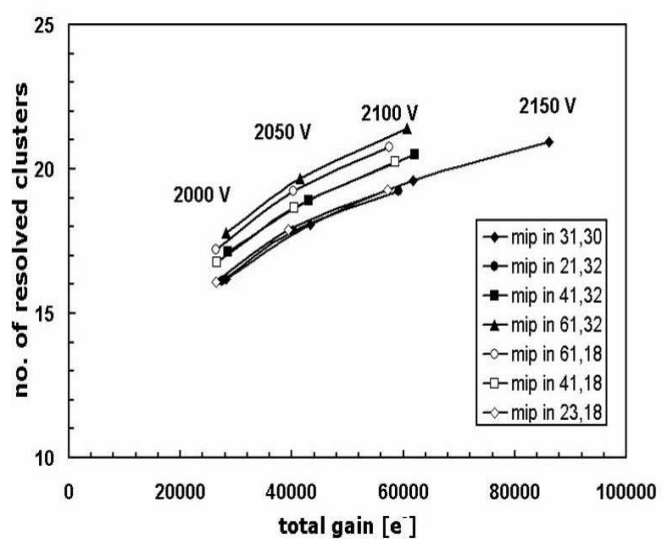

Fig. 3. The number of resolved clusters in $\mathrm{PC} 44$.

ployed to set the acceptance threshold during the mass production of the PCs [6], [8]. So far, five modules have been tested in CERN/SPS-X5 area with a $120 \mathrm{GeV} / \mathrm{c} \pi^{-}$beam at different intensities. Module-1 had been equipped with old CsI PCs ( PCs produced before starting the mass production in May '04), and was tested in 2003. Modules 2-4 have been commissioned during summer '04 with final PCs.

Raw pad clusters generated by more than one detected Cherenkov photon that overlap in the same or neighboring pads, are split in smaller pads, the so-called resolved clusters [2]. The number of resolved clusters represents the number of Cherenkov photons. For each photocathode several positions have been scanned (up to 9 different positions). For each position we took data at different gain and different beam intensities. As an example, in Fig. 3 we have the number of resolved clusters as a function of the chamber gain in PC44. Gain variations of about $5 \%$ and PC reponse variations of about $10 \%$ 
are observed over the scanned area. Fig. 4 shows the summary of the performance of the modules by means of the number of resolved clusters and the corresponding Cherenkov angle resolution measured in each photocathode for Module-2, Module-3 and Module-4. The data shown have been taking at an anode wire voltage of 2050 volts. The error bars for the full points represent the maximum and the minimum number of resolved clusters over the PC scanned area. Since the data have been taken over a long period of time (four weeks), the variations observed in the number of clusters are due to not only to variations in the $\mathrm{QE}$ of each $\mathrm{PC}$ but also to gas gain variations, and specially to $C_{6} F_{14}$ transparency, which could not be kept at the optimal level during the full data taking. The horizontal line at a value of 15 resolved clusters/event sets the acceptance threshold for the quality of the PCs. As we can see from the plot all the photocathodes above the threshold perform well in terms of Cherenkov angular resolution.

As we have discussed above, there are many parameters that determine the final number of resolved clusters apart from the QE of the PCs. Therefore, to extract the value of the quantum efficiency for each photocathode we need to evaluate them. This is done with the aid of a Monte Carlo simulation, in which all the physical processes from the Cherenkov emission in the radiator to the induction on the photocathode pad plane and the readout electronics behavior are taken into account. All the detector parameters like the transparency

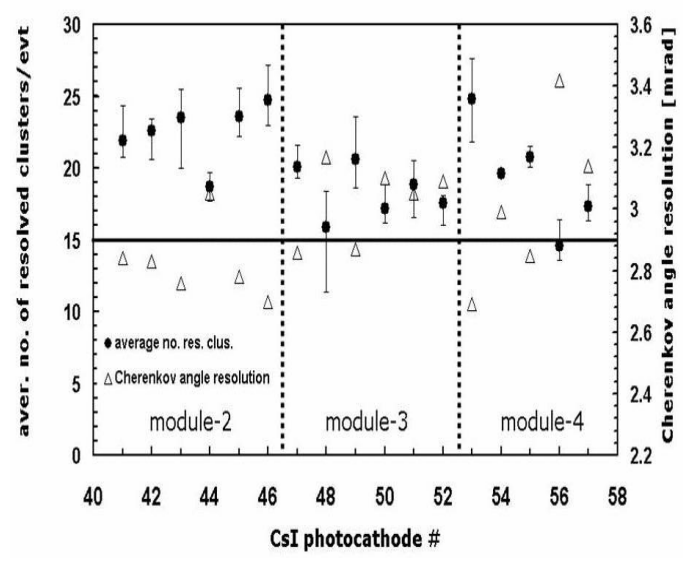

Fig. 4. Summary of the test beam results. The full points are the number of resolved clusters per event. The errors bars represent the maximum and the minimum number of resolved clusters over the photocathode scanned area. The open points are the Cherenkov angular resolution.

and refractive index of the radiator or the electronic noise are measured directly and fed into the simulation. For the QE we start with a reference curve that we vary until we reproduce the results from the test beam. Using this method we extract the QE for the PCs commissioned show them in Fig 5. A detailed description of the above procedure can be found in [10].

\section{Conclusions}

Five out of seven modules of the ALICE HMPID detector have been assembled and tested with final front end electronics in the test beam. The CsI mass production has been launched and a VUV scanner system to characterize the $\mathrm{PC}$ has been commissioned; 17 out of a total of 42 


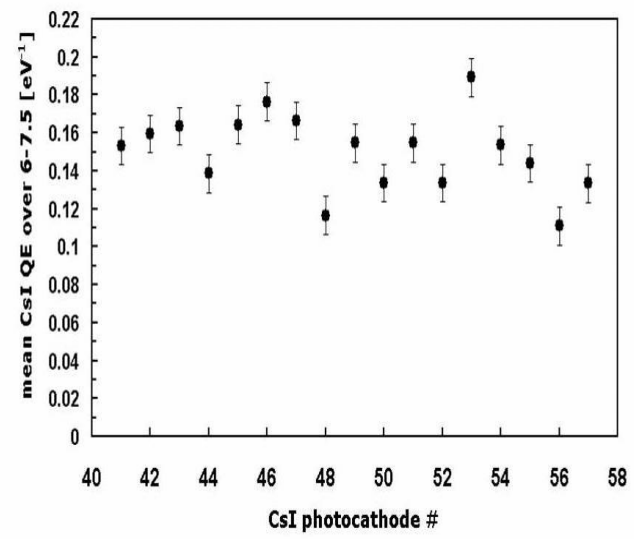

Fig. 5. Average CsI QE over 6-7.5 eV for the produced PCs.

PCs have been produced and tested. The test beam of three modules equipped with PCs from the mass production show that the detector will perform as expected. The two remaining modules will be equipped with final PCs and tested with cosmic rays before the installation in the ALICE cavern in January 2006.

\section{References}

[1] ALICE Collaboration, Physics Performance Report Vol. 1, CERN/LHCC 2003-049.

ALICE Collaboration, Technical proposal, CERN/LHCC/95-71.

[2] ALICE Collaboration, HMPID TDR, CERN/LHCC 98-19.

[3] F. Piuz et al. Nucl.Instr. and Meth. A433 (1999) 222.

[4] F. Piuz et al. Nucl.Instr. and Meth. A433 (1999) 178.

[5] E. Nappi, "The CsI-RICH detector for high momentum hadron identification in the ALICE experiment at CERNLHC", Proceedings of the XLII International Winter Meeting on Nuclear Physics, 25-31 January 2004,

Bormio, Italy - Suplemento n.123 Ricerca Scientifica ed Educazione Permanente, Università degli Studi di Milano.

[6] These proceedings, "Quality Evaluation of CsI Photocathodes for the ALICE/HMPID detector", H. Hoedlmoser et al.

[7] A. Braem et al. Nucl.Instr. and Meth. A515 (2003) 307.

A. Braem et al. Nucl.Instr. and Meth. A502 (2003) 205.

E. Schyns et al. Nucl.Instr. and Meth. A494 (2002) 441.

F. Piuz et al. Nucl.Instr. and Meth. A371 (1996) 96.

[8] A.

Di Mauro, "Status of the HMPID CsI-RICH project for ALICE at the CEN/LHC", presented at IEEE Nuclear Science Symposium, Rome 16-22 October 2004.

[9] J.C. Santiard and K. Marent, CERN-ALICE-PUB-2001-49.

J.C. Santiard, Nucl. Instr. and Meth. A518 (2004)498.

[10] A. Di Mauro et al. Nucl. Instr. and Meth. A433 (1999) 190. 